\title{
Moving magnetic tubes: fragmentation, vortex streets and the limit of the approximation of thin flux tubes
}

\author{
M. C. M. Cheung ${ }^{1}$, F. Moreno-Insertis ${ }^{2,3}$, and M. Schüssler ${ }^{1}$
}

\author{
1 Max Planck Institute for Solar System Research, 37191 Katlenburg-Lindau, Germany \\ e-mail: [cheung; msch] @mps .mpg.de \\ 2 Institúto de Astrofísica de Canarias, 38200 La Laguna (Tenerife), Spain \\ e-mail: fmi@ll.iac.es \\ 3 Dept. of Astrophysics, Faculty of Physics, University of La Laguna, 38200 La Laguna (Tenerife), Spain
}

Received 9 November 2005 / Accepted 13 January 2006

\section{ABSTRACT}

\begin{abstract}
Aims. We study the buoyant rise of magnetic flux tubes in a stratified layer over a range of Reynolds numbers $(25 \lesssim \operatorname{Re} \lesssim 2600)$ by means of numerical simulations. Special emphasis is placed on studying the fragmentation of the rising tube, its trailing wake and the formation of a vortex street in the high-Reynolds number regime. Furthermore, we evaluate the relevance of the thin flux tube approximation with regard to describing the evolution of magnetic flux tubes in the simulations.

Methods. We used the FLASH code, which has an adaptive mesh refinement (AMR) algorithm, thus allowing the simulations to be carried out at high Reynolds numbers.

Results. The evolution of the magnetic flux tube and its wake depends on the Reynolds number. At Re up to a few hundred, the wake consists of two counter-rotating vortex rolls. At higher Re, the vortex rolls break up and the shedding of flux into the wake occurs in a more intermittent fashion. The amount of flux retained by the central portion of the tube increases with the field line twist (in agreement with previous literature) and with Re. The time evolution of the twist is compatible with a homologous expansion of the tube. The motion of the central portion of the tube in the simulations is very well described by the thin flux tube model whenever the effects of flux loss or vortex forces can be neglected. If the flux tube has an initial net vorticity, it undergoes asymmetric vortex shedding. In this case, the lift force accelerates the tube in such a way that an oscillatory horizontal motion is super-imposed on the vertical rise of the tube, which leaves behind a vortex street. This last result is in accordance with previous simulations reported in the literature, which were carried out at lower Reynolds number.
\end{abstract}

Key words. magnetohydrodynamics (MHD) - Sun: magnetic fields - Sun: interior

\section{Introduction}

In order to explain solar magnetism, we need to study how magnetic fields originate in the solar interior, how they rise to the solar surface and, further, how they emerge into the atmosphere. The photospheric magnetic flux of active regions is generated in the solar interior, from where it rises in the form of buoyant magnetic tubes or bunches thereof. At present, the demand on computational resources is still far too great to carry out realistic 3-dimensional magnetohydrodynamic (MHD) simulations of a flux tube rising across the convection zone until it emerges at the photosphere, even more so if one wants to include its further rise into the upper solar atmosphere. This is due to the extremely wide range of length and time scales involved in the process and to the complicated mixture of physical phenomena associated with the rise of the tube in its different phases (e.g., turbulent convection, radiative transfer, magnetic reconnection). A possibility to make progress in spite of these limitations is to perform idealized simulations to address different aspects of the problem separately.

A branch in this undertaking is devoted to the basic magnetohydrodynamics of buoyant flux tubes rising in stratified and (otherwise) unmagnetized media studied by way of 2D or 3D numerical experiments (see review by Fan 2004). One example is an initially horizontal magnetic flux tube embedded in a stratified layer. The tube is endowed with a density deficit with respect to the surroundings, so that it rises and, in doing so, it expands, displaces the surrounding medium and develops a trailing wake. A number of results, obtained mostly for $2 \mathrm{D}$ (more precisely, 2.5D) configurations, concern the conversion of the rising magnetic tube into pairs of vortex tubes. These experiments focus on the evolution in a vertical plane normal to the axis of the tube and use the simplifying assumption of independence of all quantities (scalars or vectors) with respect 
to the coordinate along that axis. When the magnetic field in the horizontal tube is purely longitudinal (i.e., has no components in the plane normal to the tube axis), an initially cylindrical tube, after rising a height equivalent to a few times the tube diameter, splits into two roughly mirror-symmetric vortex rolls. The rolls have vorticity of opposite signs pointing in the direction of the tube axis and separate horizontally from each other. This behavior had been noted in an early paper by Schüssler (1979) and was analyzed by Longcope et al. (1996) who showed that the motion of the resulting vortex tubes could be explained as a result of the combined action of the buoyancy and lift forces on them. On the other hand, Moreno-Insertis \& Emonet (1996) demonstrated that a sufficient amount of field line twist around the tube axis can prevent the splitting of the tube: the transverse component of the field (i.e., the component in the normal plane to the tube axis) imparts rigidity to the tube interior against the stresses in their periphery. They could quantify this effect both by deriving approximate criteria and by considering the results of numerical experiments of rising tubes with different levels of twist. Moving magnetic tubes have a trailing wake with two vortex rolls in a wide range of Reynolds numbers, including those relevant for these calculations. Moreno-Insertis \& Emonet (1996) showed that the rising tube loses magnetic flux to the wake by an amount that is higher the smaller the pitch angle of the field lines around the tube axis. For an untwisted tube, the major part of the initial magnetic flux is just lost to the wake, a phenomenon that was described by the earlier authors as the conversion of the tube into two vortex rolls. Further to that, the stresses exerted on the periphery of the tube can be integrated to obtain global values for the drag and lift forces on the tube and for the added inertia of the tube caused by the co-acceleration of the surrounding plasma. Those authors showed that the motion of the tube in their experiments could be fitted reasonably using simple estimates for the drag force and enhanced inertia of the tube. Later on, Emonet \& Moreno-Insertis (1998) studied the dynamics within the vortex tube and in its surroundings and, in particular, the central role played by the magnetic and viscous boundary layers that serve as interface between them. The boundary layer is the site of generation of the vorticity that accumulates in the wake; by studying the vorticity equation it was shown that the torque of the Lorentz force is a major source of vorticity in the boundary layer. Emonet \& Moreno-Insertis (1998) also showed how the behavior of the rising tube concerning its deformation and its wake is intermediate between that of an air bubble rising in water and that of a rigid cylinder in a flow, with those two limits being reached for smaller (air bubbles) and larger (rigid cylinders) levels of twist. Hughes et al. (1998) studied in detail the effect of using different distributions of transverse field within the tube and concluded that it is the strength of the transverse field and not its distribution which is the key factor determining the coherence of the rising tube.

In a wide range of Reynolds numbers, the wake trailing rigid cylinders is unstable to vortex shedding. Shedding of eddies from the wake was observed also in rising magnetic flux tubes by Emonet \& Moreno-Insertis (1998, see their Fig. 13 and Sect. 6), Hughes \& Falle (1998) and, for a pair of rising tubes in interaction, by Fan et al. (1998a). The latter authors noted that each time a vortex roll was emitted, the head of the tube together with the remaining eddy of the wake got a net non-zero integrated vorticity, say $\boldsymbol{\Omega}$, of equal strength (but opposite sign) to the total circulation around the eddy shed. The tube therefore becomes subject to a lift force $-\rho \boldsymbol{V} \times \boldsymbol{\Omega}$, with $\boldsymbol{V}$ the forward speed of the tube. Fan et al. (1998a) explained in that way the sideways deviation of the tube trajectory away from a vertical line observed in their simulation after shedding of the eddies from the wake. In fact, as vortices were shed alternatively from the right and left side of the wake with opposite signs of the vorticity, the result is an oscillatory motion superimposed on the vertical rising trajectory for the pairs of magnetic tubes.

A further step was undertaken by Emonet et al. (2001). They studied the trajectory of a (single) flux tube initially endowed with non-zero vorticity. The vortex-shedding instability developed also in this case and led to the formation of a von Kármán vortex street behind the tube. In the simplest case, the upgoing trajectory of their tube was oscillatory, like for the pairs of tubes of Fan et al. (1998a). They attempted to understand the trajectory in a variety of cases by studying the equation of motion of a vortex filament with a time-varying value of the integrated vorticity and subject to buoyancy force, drag and lift. The solutions depend on the value of a dimensionless parameter, $\chi$, the ratio of the timescales for the drag and lift force, respectively, to modify significantly the velocity of the tube. In the drag-dominated asymptotic regime $(\chi \ll 1)$, the trajectory is a straight line which subtends a small angle $\theta \approx \sqrt{\chi}$ to the vertical and the tube moves with the terminal speed given by the equilibrium between the drag and buoyancy forces. In the drag-free (or lift- dominated) regime (i.e., $\chi \gg 1$ ), the trajectory is a cycloid with horizontal drift speed given by the ratio between buoyancy acceleration and total circulation around the tube. The intermediate cases contain rising trajectories with epicycles superimposed on them similar to features observed in the 2D simulations of Hughes \& Falle (1998).

From all the foregoing we deduce that the behavior of moving magnetic flux tubes is importantly influenced by the interaction with the surroundings via the stresses (gas pressure plus viscous and Maxwell stresses) acting at the interface between them. On the other hand, the Reynolds number in these experiments, even if still far removed from the actual astrophysical values, must be high, at least a few hundred, for the wake formation and vortex shedding phenomena described above to take place. This, in turn, makes the boundary layer thin (its width varies typically like $\propto \mathrm{Re}^{-1 / 2}$ ). So, high resolution is necessary, at least to resolve the important small-scale features that develop in the calculation. An adaptive mesh refinement (AMR) algorithm, as used by Hughes \& Falle (1998), is well suited to this end.

Calculations of that sort with adequate resolution are computationally expensive, and, as just seen, necessarily so, even though they are two-dimensional. To model the rise of magnetic tubes through the whole convection zone, typically with a view to understanding the origin of active regions and some of their global features, one has to resort to drastic simplifications. The approach commonly applied makes use of the slender (or thin) flux tube approximation (Roberts \& Webb 1978; 
Spruit 1981), which models a flux tube as a quasi-1D string of mass elements under the assumption that its diameter is small compared to any other relevant length scale of the problem. The comparatively simple $1 \mathrm{D}$ equations derived through this approximation allow to study the evolution of a flux tube in a spherical shell with a stratification taken from the mixinglength models of the solar convection zone. Using this kind of approach, it has been possible to explain the emergence latitudes of active regions, their tilt angles as well as the asymmetry between leading and following polarities (D'Silva \& Choudhuri 1991; Fan et al. 1993, 1994; Moreno-Insertis et al. 1994; Caligari et al. 1995). For details and references, the reader is referred to the reviews by Moreno-Insertis (1997) and Fan (2004). The agreement with those observations provides support for the use of the slender tube approximation in this context. However, important aspects of the flux tube dynamics, like those discussed in the previous paragraphs, are disregarded by the approximation. Furthermore, the condition of small radius compared to the local pressure scale height is not fulfilled as soon as the tubes get close to the photosphere in their rise, typically when they reach levels of about a few Mm below the surface or move above them (as in the $2.5 \mathrm{D}$ simulations of Magara 2001). So, it is important to check how the thin tube approximation performs, compared to a more exact solution, when using it close to (or even somewhat beyond) its limits of applicability.

The objective of the present paper is to carry out $2.5 \mathrm{D} \mathrm{nu}-$ merical experiments of the rise of a buoyant horizontal magnetic flux tube in a stratified medium using a state-of-the-art AMR code (the FLASH code) and profiting from the unprecedented computational power allowed by today's massively parallel computers. The article is organized as follows. In Sect. 2, we present details of the simulation setup, including the system of equations solved, the numerical method used, initial conditions of the simulation. Additionally, in Sect. 2.4, we present the method used to track the flux tube. In Sect. 3, the results from the $2.5 \mathrm{D}$ simulations are presented. The following aspects of the simulations are discussed: dependence on Reynolds number (Sect. 3.1), the dependence of the flux retention on twist (Sect. 3.2) and the evolution of the twist in the tube (Sect. 3.3). In Sect. 4, we use a thin flux tube model to explain the evolution of the flux tube as it rises through the stratified layer. In Sect. 5, we explore the transition from the thin flux tube regime to the thick flux tube regime. Finally, in Sect. 6 we discuss possible implications for understanding real magnetic flux tubes in the solar interior.

\section{Equations, numerical method and initial conditions}

\subsection{Equations}

The medium is taken to be a compressible, electrically conducting ideal gas with the equation of state:

$p=R \rho T$, where $R=R^{\star} / \bar{\mu}$ is the individual gas constant and $\bar{\mu}$ the mean molar mass. The time evolution of the system is governed by the ideal magnetohydrodynamics equations:

$$
\begin{gathered}
\frac{\partial \rho}{\partial t}+\nabla \cdot(\rho \boldsymbol{v})=0, \\
\frac{\partial(\rho \boldsymbol{v})}{\partial t}+\nabla \cdot\left(\rho \boldsymbol{v} \otimes \boldsymbol{v}-\frac{1}{\mu_{0}} \boldsymbol{B} \otimes \boldsymbol{B}\right)+\nabla p_{\mathrm{tot}}=\rho \boldsymbol{g}, \\
\frac{\partial(\rho e)}{\partial t}+\nabla \cdot\left(\boldsymbol{v}\left[\rho e+p_{\mathrm{tot}}\right]-\frac{1}{\mu_{0}} \boldsymbol{B}[\boldsymbol{v} \cdot \boldsymbol{B}]\right)=\rho \boldsymbol{g} \cdot \boldsymbol{v}, \\
\frac{\partial \boldsymbol{B}}{\partial t}+\nabla \cdot(\boldsymbol{v} \otimes \boldsymbol{B}-\boldsymbol{B} \otimes \boldsymbol{v})=0,
\end{gathered}
$$

where $\boldsymbol{v} \otimes \boldsymbol{v}, \boldsymbol{B} \otimes \boldsymbol{B}$ etc. denote dyadic products and

$$
\begin{aligned}
p_{\text {tot }} & =p+\frac{B^{2}}{2 \mu_{0}}, \\
e & =\frac{1}{2} v^{2}+\epsilon+\frac{1}{\rho} \frac{B^{2}}{2 \mu_{0}}
\end{aligned}
$$

are total pressure and total specific energy. $\epsilon$ is the specific internal energy. The solenoidal condition $\nabla \cdot \boldsymbol{B}=0$ applies as an initial condition. These equations are valid in the absence of thermal, viscous and Ohmic diffusion.

\subsection{Initial conditions}

\subsubsection{Background stratification}

A hydrostatic, adiabatically stratified polytropic layer of ideal monatomic gas was chosen as the initial background stratification. This initial polytropic layer is described by the following temperature, density and pressure profiles:

$$
\begin{aligned}
& T(y)=T_{0}\left[1+\frac{\nabla_{\mathrm{ad}}}{H_{p 0}}(d-y)\right] \\
& \rho(y)=\rho_{0}\left[1+\frac{\nabla_{\mathrm{ad}}}{H_{p 0}}(d-y)\right]^{1 / \nabla_{\mathrm{ad}}-1}, \\
& p(y)=p_{0}\left[1+\frac{\nabla_{\mathrm{ad}}}{H_{p 0}}(d-y)\right]^{1 / \nabla_{\mathrm{ad}}}
\end{aligned}
$$

where $y$ is the height coordinate, $d$ the thickness of the layer, and $T_{0}, \rho_{0}, p_{0}$ and $H_{p 0}$ are the temperature, density, gas pressure, and pressure scale height at the top boundary $(y=d)$, respectively. The logarithmic temperature gradient is $\nabla=\nabla_{\mathrm{ad}}=$ $(\mathrm{d} \ln T / \mathrm{d} \ln p)_{s}=1-1 / \gamma_{2} \cdot \gamma_{2}$ is Chandrasekhar's second adiabatic exponent (Chandrasekhar 1957). For the case of a completely ionized or completely neutral, monatomic gas, $\gamma_{2}=$ $5 / 3$. An adiabatic stratification was chosen because it is neutral to motions induced by the rise of the flux tube. This is, of course, an idealization of what happens in the solar convective zone. However, here we are interested in studying the rise of the tube purely by its own buoyancy. Furthermore, for $B \gg B_{\mathrm{eq}}$, where $B_{\text {eq }}$ is the equipartition field strength with respect to the 
convective flows, we can safely neglect the effects of the external convection on the rise of the tube.

We choose to use $\rho_{0}, p_{0}$ and $H_{p 0}$ as units for the density, pressure and length respectively. $R T$ is used as the temperature variable. The units for the velocity and time are $c_{0}=\sqrt{p_{0} / \rho_{0}}$ (the isothermal sound speed at the top of the layer) and $t_{0}=$ $H_{p 0} / c_{0}$ respectively. The unit for specific energies is $e_{0}=p_{0} / \rho_{0}$ and the unit for the magnetic field is $B_{0}=\sqrt{2 \mu_{0} p_{0}}$. Expressed in these units, the initial polytropic profiles (8) to (10) become

$$
\begin{aligned}
& \tilde{T}(\tilde{y})=\left[1+(\tilde{d}-\tilde{y}) \nabla_{\mathrm{ad}}\right], \\
& \tilde{\rho}(\tilde{y})=\left[1+(\tilde{d}-\tilde{y}) \nabla_{\mathrm{ad}}\right]^{1 / \nabla_{\mathrm{ad}}-1}, \\
& \tilde{p}(\tilde{y})=\left[1+(\tilde{d}-\tilde{y}) \nabla_{\mathrm{ad}}\right]^{1 / \nabla_{\mathrm{ad}}},
\end{aligned}
$$

where the tilde denotes a quantity expressed in these units. In what follows, all quantities are expressed in terms of these units and we drop the tilde from all the symbols. Quantities inside the flux tube are denoted with a subscript $i$ and quantities outside the flux tube have no subscripts. For example, $T$ and $T_{\mathrm{i}}$ refer to the temperature outside and inside the flux tube respectively. From here on, the symbol $H_{p}$ always refers to the local pressure scale height.

In the simulations presented in Sect. 3, the polytropic layer is enclosed in the region $x \in[-10,10]$ and $y \in[0,35]$. Therefore the density and pressure contrasts between the bottom and top of the polytropic layer are 58 and 871 respectively. The number of pressure scale heights spanned over the height of the layer is $N_{p}=\int \mathrm{d} y / H_{p}=6.77$. This is comparable to the number of pressure scale heights spanned between the bottom of the solar convection zone (at depth of $200 \mathrm{Mm}$ ) and a depth of about $20 \mathrm{Mm}$. Thin flux tube simulations of flux tubes carrying magnetic flux comparable to active regions $\left(\Phi=10^{20}-10^{22} \mathrm{Mx}\right)$ are considered to yield reliable results up to a depth of about $10 \mathrm{Mm}$. Above that depth, the radii of these flux tubes become comparable to the local $H_{p}$. The number of pressure scale heights between a depth of $200 \mathrm{Mm}$ to $10 \mathrm{Mm}$ is $N_{p} \approx 16$.

\subsubsection{Initial magnetic profile of the flux tube}

The initial flux tube is taken to be axisymmetric. In cylindrical coordinates, the longitudinal and azimuthal components of its magnetic field have the form:

$$
\begin{aligned}
& B_{l}(r)=B_{0} \exp \left(-r^{2} / R_{0}^{2}\right), \\
& B_{\theta}(r)=\frac{\lambda r}{R_{0}} B_{l}
\end{aligned}
$$

where $r \in\left[0,2 R_{0}\right]$ is the radial distance from the tube axis, $R_{0}$ is the characteristic radius of the flux tube and $\lambda$ is the dimensionless twist parameter. For $r>2 R_{0}$, the magnetic field is zero. As such, a twisted magnetic flux tube of this form carries no net current. We note that the parameter $q$ used by Linton et al. (1996) and Fan et al. (1998b) is equivalent to $\lambda / R_{0}$. At $r=R_{0}$, the pitch angle is given by $\tan \Psi=\left.\left(B_{\theta} / B_{l}\right)\right|_{R_{0}}$. The total initial longitudinal flux of the tube is $\int B_{l} \mathrm{~d} A=0.98 \pi R_{0}^{2} B_{0}$.
For each simulation, a flux tube was inserted near the bottom of the stratified layer at time $t=0$. We have carried out a number of simulations with different values of $R_{0}$ and $\lambda$.

\subsection{Numerical method}

We used the FLASH code to carry out the simulations. This code implements a Riemann solver that is formally second-order accurate in time and space. The advective terms are discretized using a slope-limited Total Variation Diminishing (TVD) scheme and time-stepping is performed using an explicit, Hancock-type scheme (Toro 1997). The system of equations is solved on a $2 \mathrm{D}$ grid with Cartesian geometry.

As mentioned in Sect. 2.1, the MHD Eqs. (2)-(5) do not take into account the effects of thermal, viscous and Ohmic diffusion. In practice, however, such non-ideal effects are always present in simulations as a result of numerical diffusion. Of course, if diffusive effects are actually important for the problem of interest, diffusive terms can be added to the MHD equations to capture the relevant physics. In astrophysical problems, one often encounters situations with very large dynamic and magnetic Reynolds numbers. This is also the case for the solar interior. As such, we have chosen not to impose explicit diffusive terms in the MHD equations. Diffusive effects in the following simulations are purely numerical. The amount of numerical diffusion present in the simulation is dependent on the numerical resolution used. The higher the numerical resolution, the smaller is the amount of numerical diffusion and the larger are the effective Reynolds numbers. Since the initial state of the background atmosphere in our problem is uniform (except for small pressure perturbations) in the horizontal direction and smoothly varying in the vertical direction, only relatively large grid spacing is required to resolve regions far away from the flux tube. On the other hand, high spatial resolution is needed to resolve the small-scale features at the interface between the tube and its surroundings (e.g., in regions where the flux tube fragmented).

The numerical resolution we can use is limited by the computational resources available. To get the highest numerical resolution where we need it, we made use of the Adaptive Mesh Refinement (AMR) feature in FLASH. The Cartesian domain is comprised of adjacent square blocks, each consisting of $8 \times 8$ grid cells. At each time-step, all the blocks are checked to determine if the block should be refined. If the normalized second-order spatial derivative of the absolute field strength, $|B|$, exceeds some fixed threshold in any grid cell, the resolution of the corresponding block is doubled by interpolation and the original block is split into four sub-blocks, increasing the "refinement level" of the original block by one. The reverse process (coarsening) occurs when the normalized secondorder spatial derivative of $|B|$ is smaller than some threshold for all four sub-blocks. Then the resolution of each sub-block is halved and the sub-blocks are merged.

For further details on the FLASH code, the reader is referred to the FLASH user manual (ASCI/ Alliance Center for Thermonuclear Flashes 2003). 


\subsection{Tracking the flux tube}

In the simulations discussed here, the initial flux tube does not remain a single, monolithic structure as it rises to the top. From the results of the literature (Emonet \& Moreno-Insertis 1998; Fan et al. 1998a; Hughes \& Falle 1998), we expect the tube to fragment and lose flux by means of vortex shedding. However, for a sufficiently high level of field line twist, a central portion of the tube retains its identity throughout the simulation. We refer to this central flux filament as the "main tube". To track the main tube, we make use of the flux function:

$\psi(x, y)=\int_{0}^{x} B_{y}\left(x^{\prime}, y\right) \mathrm{d} x^{\prime}-\int_{0}^{y} B_{x}\left(x, y^{\prime}\right) \mathrm{d} y^{\prime}$.

Field lines traced out by the transverse field $\left(B_{x}, B_{y}\right)$, when projected onto the $x-y$ plane, correspond to contours of $\psi$. For the initial axisymmetric flux tube, the contours are a family of concentric circles corresponding to the planar projection of the twisted field lines that wind around the tube axis. Let us call one of these circles $C$. The initial magnetic flux inside this structure is given by $\Phi_{0}=\int_{C} B_{z} \mathrm{~d} A$. To identify this structure at a later time, we calculate $\psi$ for that time and find the contour(s) $\partial C^{\prime}$ satisfying the flux conservation criterion $\Phi_{t}=\int_{C^{\prime}} B_{z} \mathrm{~d} A=\Phi_{0}$. If the structure has broken up, then the contours will be a set of closed curves and the sum of the fluxes enclosed in these curves is $\Phi_{0}$.

If we arbitrarily chose a value of $\Phi_{0}$ to define the main tube, we have no guarantee that at a later time, the structure we track remains coherent. In order to define a coherent main tube, we reverse the aforementioned procedure. At the end of a simulation (i.e. when the main flux structure has reached the top of the domain), $t=t_{1}$, we calculate $\left.\psi(x, y)\right|_{t 1}$. We test different contour levels $\psi_{0}=\left.\psi\right|_{t 1}$. If the contours corresponding to a particular value of $\psi_{0}$ consists of more than one closed loop, we dismiss them. For the remaining values of $\psi_{0}$ (each of which has only one corresponding closed curve), we pick the one that encloses the maximum amount of flux $\left(\Phi_{\mathrm{MT}}\right)$. This is defined as the main tube for this particular simulation. To back-track the main tube at an earlier time, we simply calculate $\psi$ for that time. Then the contour which encloses a flux equal to $\Phi_{\mathrm{MT}}$ represents the main tube at that time.

The centre of the main tube is located at the extremum of $\psi$ (maximum or minimum depending on whether the tube has right or left-handed twist). Although the main tube is, in general, not circular, we can define an effective radius,

$r_{\text {eff }}=\sqrt{\int_{C} \mathrm{~d} A / \pi}$,

which gives a useful measure of its size.

\section{Simulation results}

We have carried out a number of simulations in order to study different aspects of the problem of the buoyant rise of magnetic flux tubes. Various aspects of the results are discussed in the following sections.
Table 1. Simulation runs carried out to study the dependence of the simulation result on numerical resolution (and hence Reynolds number).

\begin{tabular}{rcc}
\hline \hline Run & Effective resolution & Re \\
\hline A1 & $256 \times 448$ & 25 \\
A2 & $512 \times 896$ & 140 \\
A3 & $1024 \times 1792$ & 630 \\
A4 & $2048 \times 3584$ & 2600 \\
\hline
\end{tabular}

\subsection{Dependence on Reynolds number}

From the same initial setup, we have carried out simulations with different levels of grid refinement in order to study how the numerical resolution influences the outcome of the simulation. The initial condition is as follows: a flux tube was inserted near the bottom of the polytropic atmosphere at $\left(x_{0}, y_{0}\right)=(0.0,2.5)$ at $t=0$. The flux tube has $R_{0}=0.5$ (corresponding to $4 \%$ of the local pressure scale height), $\beta_{0}=15$ and $\lambda=0.25$. The material inside the tube has the same entropy as the external atmosphere, so that it is buoyant. We carried out four runs from this initial condition. Run A1 has the lowest effective resolution. If the simulation domain was fully refined, the domain would be spanned by $256 \times 448$ grid cells in the $x$ and $y$ directions. Runs A2, A3 and A4 have 2, 4 and 8 times the effective resolution of A1 respectively. Table 1 gives the effective resolution and effective Reynolds numbers $(\mathrm{Re})$ for each of the runs. The latter is defined as

$\operatorname{Re}=\left(\frac{D}{L_{\mathrm{bl}}}\right)^{2}$,

where $D$ is the flux tube diameter and $L_{\mathrm{bl}}$ is the width of the boundary layer between flux tube and its surroundings.

Figure 1 shows the distribution of the longitudinal field $\left(B_{z}\right)$ over the entire domain at $t=200$ for all four runs. Figure 2 shows the $z$ component of the vorticity $\left(\omega_{z}\right)$ at the same time. To emphasize the difference in resolution between the runs, the axes are labelled in terms of grid-points. To calculate Re, we examined the profiles of $B_{z}$ and $\omega_{z}$ near the vertical $x=0$ for each of the runs at $t=200$. From the $B_{z}$ profile we can find the tube diameter $D$. From the corresponding $\omega_{z}$ profile, we identify a thin boundary layer near the apex of the tube. The left and right halves (about $x=0$ ) of this boundary layer have opposite sign. The thickness of this boundary layer - which is the site of vorticity generation - corresponds to $L_{\mathrm{bl}}$. Inspection of the vertical profile of the magnetic field along $x=0$ gives the distance over which the magnetic field goes to zero above the tube apex. This gives the thickness of the magnetic boundary layer. For the simulations in this study, we found that the magnetic and viscous boundary layers have similar thickness, about 6 grid cells, indicating that the magnetic Reynolds number $\mathrm{Re}_{\mathrm{m}} \sim \mathrm{Re}$. This is not a coincidence, since the viscous and magnetic diffusion stem from diffusion inherent in the numerical scheme. 

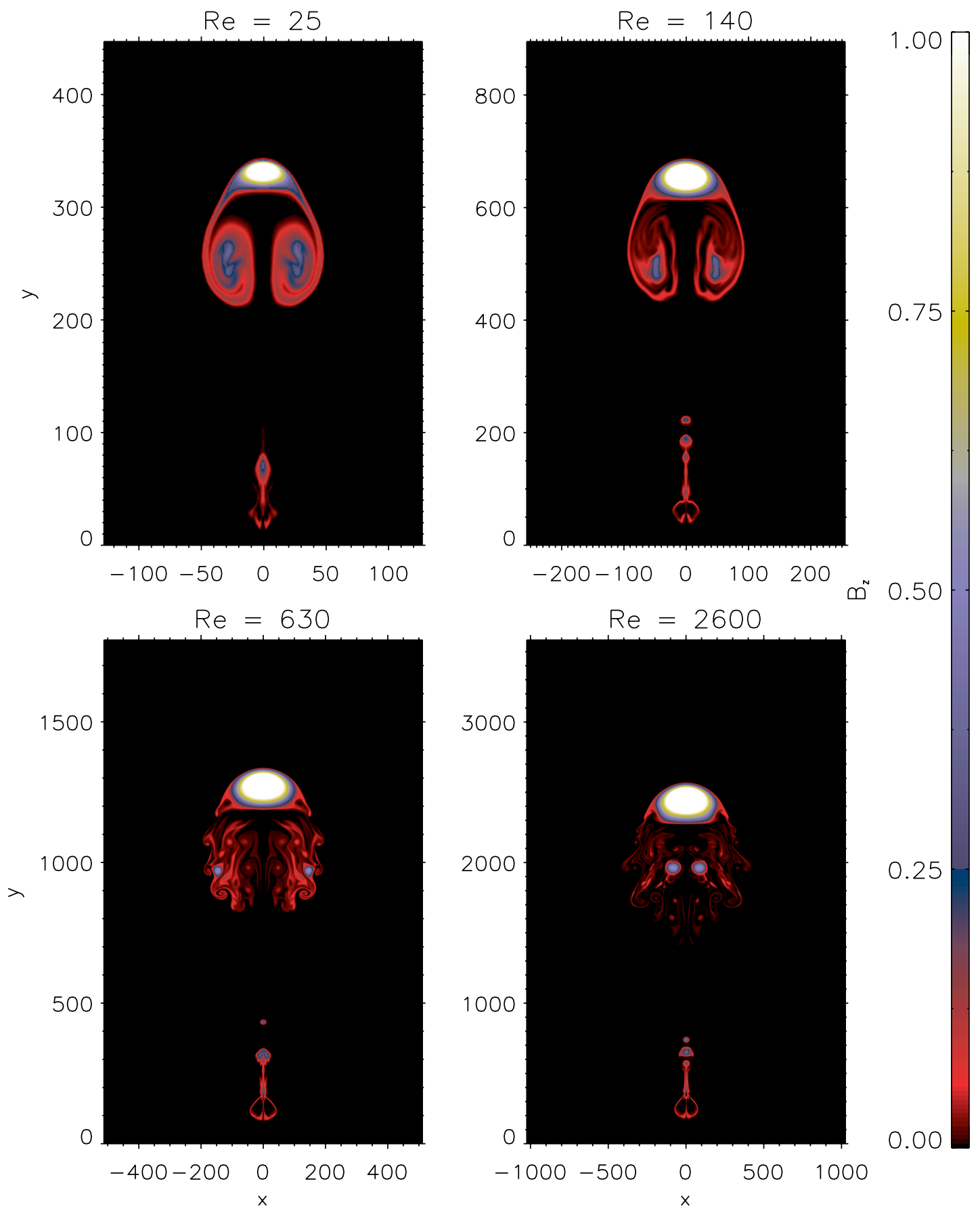

Fig. 1. The structure of the wake below the rising flux tube depends on the Reynolds number of the flow. The four panels show the distribution of the longitudinal magnetic field at Reynolds numbers ranging from 25 to 2600.

\subsubsection{Structure of the wake}

The structure of the wake depends on the Reynolds number. At $\mathrm{Re}=25$ (top left panel of Figs. 1 and 2), we identify two coherent counter-rotating vortex rolls in the wake. These types of wake structures have been reported in past studies of rising magnetic flux tubes (Schüssler 1979; Longcope et al. 1996; Moreno-Insertis \& Emonet 1996; Emonet \& MorenoInsertis 1998). A similar pair of vortex rolls is also found in the wake of the flux tube for the run with $\mathrm{Re}=140$. In this case, however, the rolls have more internal structure. Figure 9 of Emonet \& Moreno-Insertis (1998) shows similar plots of vorticity for a rising twisted flux tube. We note that the vorticity distribution shown in the rightmost panel of their figure very much resembles our case for $\operatorname{Re}=140$. However, the vortex rolls in their figure also show signs of breakup into smaller vortex rolls. At higher $\operatorname{Re}(\operatorname{Re}=630$ and $\mathrm{Re}=2600$ in runs $\mathrm{A} 3$ and A4 respectively), the breakup of the vortex rolls is even more obvious. In these two cases, each roll is replaced by a group of secondary rolls with the same sign in vorticity. This indicates that the shedding of material at high Reynolds number occurs in a much more intermittent fashion than in cases with low Re. 

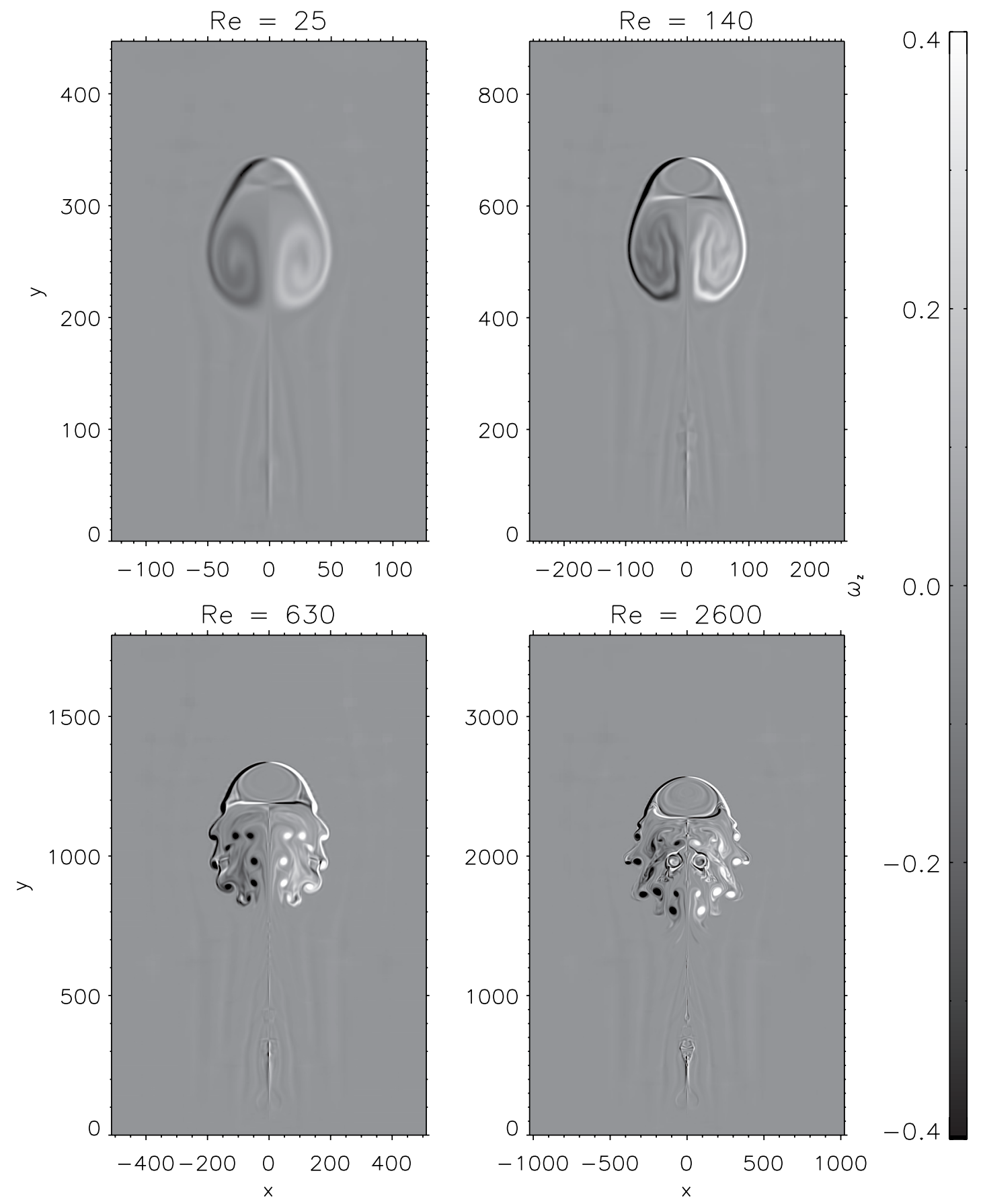

Fig. 2. Same as Fig. 1 but for the $z$ component of the vorticity. At low Reynolds numbers - see cases with $\operatorname{Re}=25$ and $\operatorname{Re}=140-$ the wake consists of two vortex rolls with vorticity of opposite sign. At sufficiently high Reynolds numbers - see cases with $\operatorname{Re}=630$ and $\operatorname{Re}=2600-$ the vortex rolls break up and the shedding of vorticity into the wake occurs in a more intermittent fashion.

\subsubsection{Flux retention and field diffusion}

The fraction of magnetic flux retained by the head of the flux tube (i.e. the main tube) also depends on the Reynolds number. Figure 3 shows the flux retained in the main tube at $t=280$ for the four different runs (diamonds). Clearly, with increasing $\mathrm{Re}$, the percentage of flux retained by the main tube increases. At $\mathrm{Re}=2600$, the main tube retains $84 \%$ of the original flux of the initial tube. Although we cannot conclude from these simulations that the flux retained converges to some value in the limit $\operatorname{Re} \rightarrow \infty$, Fig. 3 does seems to suggest that the curve levels off for increasing Re.

Emonet \& Moreno-Insertis (1998) demonstrated that in a rising, twisted flux tube, vorticity is generated in the magnetic boundary layer between the tube and the surrounding flow. The material in this boundary is then advected towards the wake, leading to a loss of magnetic flux from the tube. We can estimate the flux loss per unit time as

$-\frac{\mathrm{d} \Phi}{\mathrm{d} t}=v_{\mathrm{tube}} L_{\mathrm{bl}} B_{\mathrm{bl}}$ 


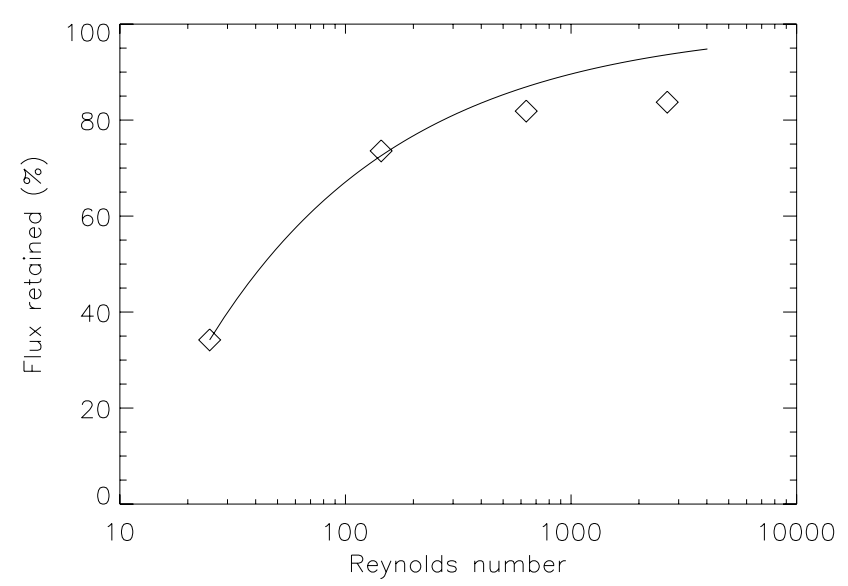

Fig. 3. Magnetic flux retained in the main tube as a function of the effective Reynolds number. The diamonds plot the values from simulations $\mathrm{A} 1$ to $\mathrm{A} 4$. If the amount of flux lost scaled as $O\left(\mathrm{Re}^{-1 / 2}\right)$, it would follow the solid curve.

where $v_{\text {tube }}$ is the relative velocity between the tube and the surroundings and $B_{\mathrm{bl}}$ is the characteristic value of the longitudinal field in the boundary layer. Making use of Eq. (18), and using the approximation that $\mathrm{Re} \approx \mathrm{Re}_{\mathrm{m}}$, we find that

$-\frac{\mathrm{d} \Phi}{\mathrm{d} t}=v_{\mathrm{tube}} B_{\mathrm{bl}} \frac{D}{\sqrt{\mathrm{Re}}}$

If one makes the additional assumptions that, $v_{\text {tube }}, D$ and $B_{\mathrm{bl}}$ do not change much with $\mathrm{Re}$, then we find that the amount of flux lost from the tube scales as $O\left(\mathrm{Re}^{-1 / 2}\right)$. The amount of flux retained by the main tube, as predicted by this scaling relation, is plotted as a solid line in Fig. 3. The value at $\mathrm{Re}=25$ was used as a reference point for the curve.

In ideal MHD, the ratio of mass and longitudinal magnetic flux enclosed in the main tube, $M / \Phi$, should remain constant. In numerical simulations, however, the ratio always increases with time because of some mass diffusion across field lines. The size of this change tells us how well the simulation approximates the ideal MHD case. Figure 4 shows the percentage change of this quantity for the main tube between $t=0$ and $t=280$, as a function of $\mathrm{Re}$. At $\operatorname{Re}=25$, the ratio $M / \Phi$ increased by $44 \%$. This increase in the ratio diminishes for higher resolution. At $\operatorname{Re}=2600$, the change is only on the order of $1 \%$. Consequently, in order to compare the results from numerical simulations with predictions in the approximation of thin flux tubes (which assumes ideal MHD), we should take the results from runs with the highest values of Re.

\subsection{Dependence of flux retention on twist}

We carried out a number of simulations to study the dependence of the flux retained in the main tube as a function of the twist parameter $\lambda$. These runs have the same resolution as run $\mathrm{A} 4$. We find that a flux tube with no initial twist $(\lambda=0)$, after rising a distance a few times its radius, splits into two counter-rotating vortex rolls, which separate horizontally from each other. This behaviour has already been reported in an early paper by Schüssler (1979). Longcope et al. (1996) showed that this is a result of the combination of the buoyancy and lift

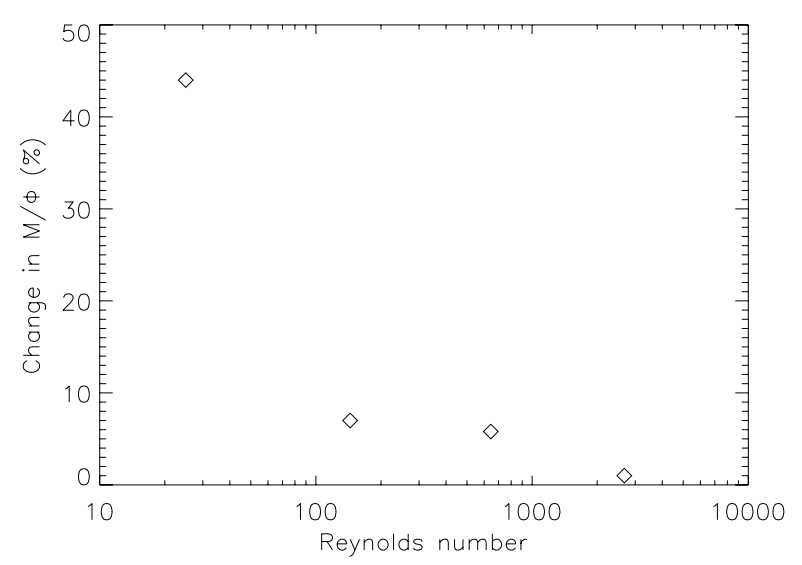

Fig. 4. The change of $M / \Phi$ (ratio of enclosed mass and enclosed magnetic flux in the main tube) as a function of the Reynolds number.

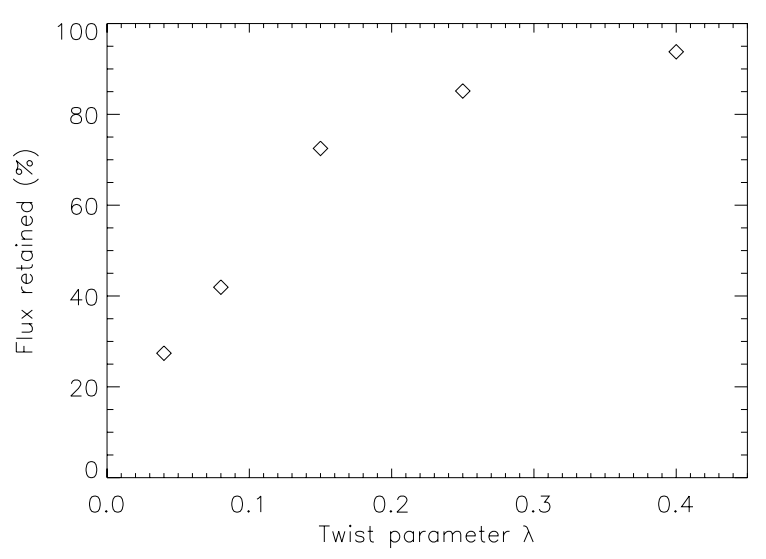

Fig. 5. The dependence of the fraction of flux retained in the main tube as a function of the twist parameter $\lambda$.

forces acting on the flux tube. Our results show that even at the relatively high Reynolds number $\left(\operatorname{Re} \sim 10^{3}\right)$, the same behaviour is observed.

For magnetic flux tubes with non-zero twist, we were able to track a main tube. Figure 5 shows the amount of flux retained in the main tube at $t=280$. It is a monotonically increasing function of $\lambda$, a result consistent with the previous work of Moreno-Insertis \& Emonet (1996). Thus, given that a main tube can be tracked, the amount of flux it retains increases with Re.

\subsection{Evolution of twist in the flux tube}

As Parker $(1974,1979)$ pointed out, the radial expansion (compression) of a twisted flux tube leads to an increase (decrease) of the pitch angle of the field lines. This is a consequence of magnetic flux conservation (Fan et al. 1998b).

The radial profiles of the longitudinal and transverse field we have chosen for the initial flux tube yield a pitch angle of the field lines that depends on radial distance from the axis. A more appropriate measure for the amount of twist in the tube is $\lambda$, which is dimensionless and constant over the initial tube. If the 


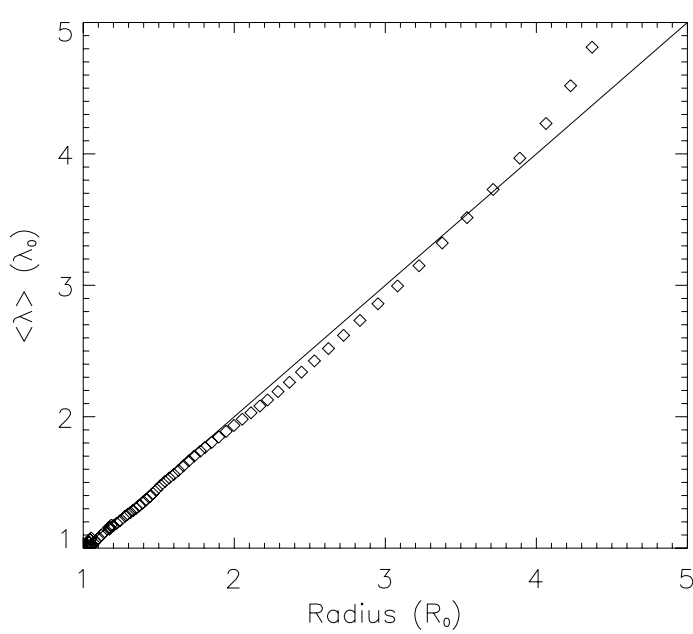

Fig. 6. The relationship between the tube's effective radius and its twist $\lambda$. The solid line shows the linear relation given by Eq. (21). Diamonds denote the evolution of the mean twist $\langle\lambda\rangle$ of the main tube in run A4. This good match indicates that the main tube expands homologously over most of the tube rise.

flux tube undergoes a homologous expansion (or compression), $\lambda$ evolves according to

$\frac{\lambda}{\lambda_{0}}=\frac{R}{R_{0}}$,

where the $\lambda$ and $R$ are the twist and characteristic radius of the tube, respectively. The subscript 0 denotes their initial values. Equation (21) states that $q=\lambda / R$ remains constant as the tube rises (Linton et al. 1996). The second-order thin flux tube approximation also predicts the same result (Ferriz-Mas et al. 1989). In this approximation, an axisymmetric tube has a transverse field $B_{\theta}(r) \propto r$. By virtue of the conservation of the transverse magnetic flux, Eq. (21) can be obtained.

In Fig. 6, the relationship given by this equation is shown as a solid line. Overplotted (as diamonds) are values of the mean twist $\langle\lambda\rangle$ of the main tube in run A4 against its effective radius $r_{\text {eff }}$. Following (15), we define the mean twist as

$\langle\lambda\rangle=r_{\mathrm{eff}}\left\langle\frac{B_{\theta}}{r^{\prime} B_{z}}\right\rangle$,

where $r^{\prime}$ is the distance of a point in the main tube from the tube centre. The match between the simulation results and the linear law is very good up to $R \simeq 3.6 R_{0}$, indicating good compatibility with the assumption of homologous expansion. Beyond this size, the flux tube radius is larger than the local pressure scale height and there is a clear deviation between the linear law and the simulation results.

\section{Comparison with a thin flux tube model}

One of the main aims of this paper is to evaluate the relevance of the thin flux tube approximation with regards to describing the behavior of flux tubes in $2.5 \mathrm{D}$ simulations. In the following, we consider how a rising magnetic flux tube behaves in the context of this approximation (Roberts \& Webb 1978; Spruit 1981).

\subsection{Thin flux tube model}

The basic assumption of the thin flux tube approximation is that the radius of the flux tube is much smaller than any other characteristic length scale in the system (e.g., local pressure scale height and the radius of curvature of the tube axis). We assume that the quantities are uniform over the tube cross-section, so that their values at the tube axis are representative of their offaxis values. This assumption corresponds to retaining only the zeroth-order term in the axis-centered Taylor-expansion of the quantities in the tube. Higher-order treatments can also be derived (Roberts \& Webb 1978; Ferriz-Mas et al. 1989). In the following, we develop a model based on the zeroth-order approximation, which is already sufficient for modelling how the physical properties near the tube centre evolve. To model the evolution of the twist in the main tube, it is necessary to extend to a second-order approach.

Instantaneous pressure balance $\left(p_{\mathrm{ex}}=p_{\text {in }}+B^{2} / 2 \mu_{0}\right)$ between the tube and its surroundings is assumed. For this assumption to hold, we require that the sound-crossing time over the tube diameter be much smaller than the time required for the tube to transverse a distance comparable to its diameter. This means the adiabatic sound speed $c_{\mathrm{S}}$ is much larger than the speed of the tube. Taking the terminal velocity (Parker 1975; Moreno-Insertis \& Emonet 1996):

$v_{\text {term }}=\sqrt{\frac{\pi}{C_{D}} \frac{\Delta \rho}{\rho} g R}$

as the characteristic speed of the tube, and taking $|\Delta \rho / \rho| \approx 1 / \beta$, we arrive at the criterion:

$\left(\frac{R_{\text {tube }}}{H_{p}}\right)^{1 / 2}\left(\frac{1}{\beta}\right)^{1 / 2} \ll O(1)$.

We make the additional assumption that the horizontal thin flux tube evolves adiabatically, subject to instantaneous pressure balance with its surrounding. This means that the state of the tube at any time is only a function of its initial state and the height it has risen. A convenient measure for the height of the flux tube is the external pressure contrast,

$\chi_{p} \equiv p_{1} / p_{0}$,

where $p_{0}$ is the ambient pressure at the initial height of the tube and $p_{1}$ is the ambient pressure at a different height. Thus, the physical quantities within the flux tube (e.g. $\beta, \rho_{i}$ etc.) at any time are functions of their initial values and $\chi_{p}$.

For a uniform horizontal flux tube, conservation of longitudinal magnetic flux leads to

$\frac{\rho_{i 1}}{\rho_{i 0}}=\frac{B_{1}}{B_{0}}$

where $B$ here is the longitudinal field strength of the thin flux tube. Combining this with the condition of instantaneous pressure balance, we have

$\frac{\rho_{i 1}}{\rho_{i 0}}=\chi_{p}^{1 / 2}\left(\frac{\beta_{0}+1}{\beta_{1}+1}\right)^{1 / 2}$. 
From the adiabatic evolution of the flux tube,

$\frac{\rho_{i 1}}{\rho_{i 0}}=\left(\frac{p_{1 i}}{p_{0 i}}\right)^{1 / \gamma}$,

and Eq. (27), we obtain

$\frac{\beta_{1}+1}{\beta_{0}+1}=\left(\frac{\beta_{1}}{\beta_{0}}\right)^{2 /(2-\gamma)} \chi_{p}$.

Given an initial value $\beta_{0}$, we can solve for $\beta_{1}$, after the tube has risen through a pressure contrast of $\chi_{p}$. For $\beta \gg 1$, we have $\beta_{1} / \beta_{0}=\chi_{p}^{(\gamma-2) / \gamma}$. The physical properties of the flux tube can be expressed as functions of $\beta_{1}$ in the following way

$$
\begin{aligned}
& B\left(\chi_{p}\right)=B_{0}\left(\frac{\beta_{1}}{\beta_{0}}\right)^{1 /(\gamma-2)}, \\
& \rho_{i}\left(\chi_{p}\right)=\rho_{i 0}\left(\frac{\beta_{1}}{\beta_{0}}\right)^{1 /(\gamma-2)}, \\
& T_{i}\left(\chi_{p}\right)=T_{i 0}\left(\frac{\beta_{1}}{\beta_{0}}\right)^{(\gamma-1) /(\gamma-2)}, \\
& R\left(\chi_{p}\right)=R_{0}\left(\frac{\beta_{1}}{\beta_{0}}\right)^{1 /(4-2 \gamma)},
\end{aligned}
$$

where $R$ is the radius of the flux tube and the expressions on the r.h.s. can be expressed in terms of $\chi_{p}$ by virtue of Eq. (29).

Of the four simulation runs A1 to A4, we chose to compare the thin flux tube model with results from Run A4 because the effect of magnetic diffusion is smallest for this case. Figure 7 shows the dependence of $|B|, T, \beta$ and the tube radius as functions of $\chi_{p}$. The values of $|B|, T$ and $\beta$ in the simulation were taken at the centre of the main tube, and are plotted as diamonds. The effective radius $r_{\text {eff }}$ of the main tube is defined by Eq. (17). The solid lines show these quantities as calculated with Eqs. (29) to (33). Since $\chi_{p}$ is the pressure contrast, $\chi_{p}<1$ corresponds heights above the original position of the tube. For this simulation, we have tracked the main tube until $\chi_{p}=5.4 \times 10^{-3}$, corresponding to over 5 pressure scale heights. The thin tube predictions agree well with the simulation results over this wide range of heights, even at the lowest values of $\chi_{p}$ where the radius becomes comparable to or larger than the local pressure scale height. The thin flux tube predictions of temperature, density and $|B|$ deviate from their actual values in the main tube centre by less than $2 \%$. The values of $\beta$ calculated with the thin tube model deviate from the actual values by $3 \%$ at most and the effective radius of the main tube differs from the theoretical value by less than $4 \%$.

The comparison we have made here shows that Eqs. (29) to (33) accurately describe the height dependence of the physical properties in the tube centre. In order for our thin tube model to be a dynamical model, we must also solve the equation of motion for a thin flux tube. This will then allow us to model the motion of the main tube as well as the timedependence of its physical properties in the thin flux tube framework. This comparison is carried out in the following section.
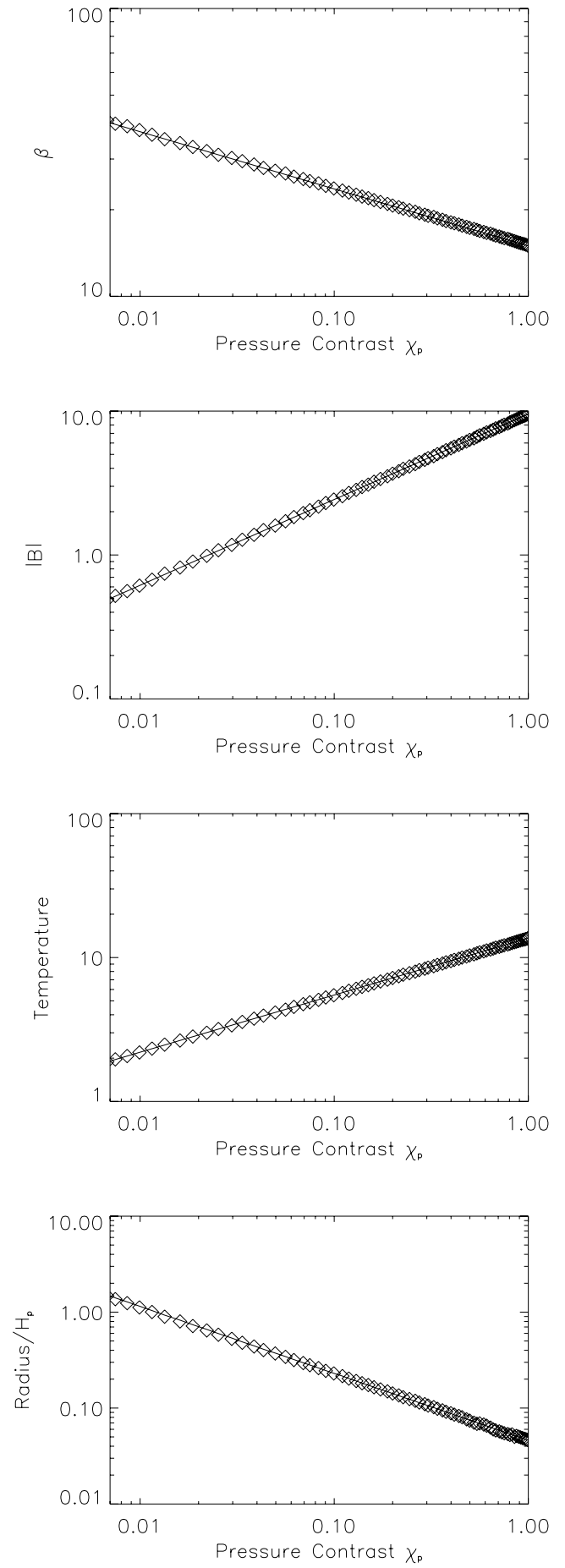

Fig. 7. Comparison between the simulation (run A4) and the thin flux tube model. Diamonds indicate values of the physical quantities at the tube centre in the simulation and the solid lines show the predictions from the thin tube model (Eqs. (29)-(33)).

\subsection{Modelling the motion of the main tube}

As explained in the introduction, the motion of a twisted magnetic flux tube in an unmagnetized environment shares a number of features with the motion of a rigid cylinder in a flow (Emonet \& Moreno-Insertis 1998; Emonet et al. 2001). Under a number of simplifying assumptions, the equation of motion of the magnetic tube can be written in a simple way; among them 
we count: (1) zero circulation and small Mach number of the surrounding flow; (2) not too low Reynolds numbers ( $\operatorname{Re} \gtrsim 50$ ); (3) small length-scales and long timescales of change for the flux tube compared to the intrinsic length and time scales, respectively, of the flow. Under those assumptions, the integrated effect of the fluid stresses on the periphery of the tube can be simply described by a drag force given by the classical aerodynamic formula and an enhancement of the inertia of the tube because of the co-acceleration of the external medium in the vicinity of the tube (Batchelor 1967). For a cylinder or flux tube driven by its own buoyancy in rectilinear motion this would yield:

$I \dot{v}_{y}=g \frac{\Delta \rho}{\rho}-\frac{C_{D}}{\pi} \frac{\left|v_{y}\right| v_{y}}{R}$,

where $v_{y}$ is the vertical velocity of the tube, $\Delta \rho / \rho$ the relative density difference between the tube and its environment and $g$ the gravitational acceleration. The second term on the right is the drag acceleration, with $C_{D}$ being the aerodynamic drag coefficient (of order unity for $\operatorname{Re} \gg 1$ ) and $I$ the enhanced inertia factor (which is $\approx 2$ for $\beta \gg 1$ ).

Figure 8 shows the rise velocity of the main tube (upper panel) and its height (lower panel), both as functions of time. Values from the simulation are indicated as diamonds. The solid lines indicates the theoretical profiles found by integrating Eq. (34). The mean density deficit of the main tube, $\langle\Delta \rho / \rho\rangle=0.013$, and its initial radius, $R=0.64$, were used as initial conditions for the path integration. The values of the drag coefficient and enhanced inertia factor used are $C_{D}=2.0$ and $I=2.0$ respectively. To take into account the effect of tube expansion on the buoyancy and drag forces, Eqs. (31) and (33) were used to update the tube radius and density at each time step of the path integration.

The velocity and height profiles from the thin tube approximation are in general agreement with the motion of the main tube between $t=0$ and $t=200$. Between $t=0$ and $t=20$, the main tube approximately undergoes a free-fall acceleration in accordance with its own buoyancy. The motion of the main tube during this time interval is well matched by the solid lines. The time taken for the main tube to rise a height difference of $\Delta y=30$ (corresponding to 4.1 pressure scale heights) is $\Delta t=273$. The corresponding rise time predicted by the thin flux tube mode is $\Delta t=256$, which is within $10 \%$ of the actual value.

One feature of the motion of the main tube which is not predicted by the thin tube calculations is the temporary deceleration of the tube between $t=20$ and $t=30$. As reported by Moreno-Insertis \& Emonet (1996), this is a result of the differential acceleration experienced by the different parts of the flux tube. Since, initially, the core of the tube has the largest density deficit, it undergoes a stronger acceleration than the parts of the tube above it. This differential acceleration leads to a compression at the apex of the tube, which enhances the transverse magnetic field there. The enhanced magnetic tension near the apex of the tube eventually decelerates the core of the flux tube (our main tube) and induces an internal oscillation.

This effect, which is not described by Eq. (34) for the motion of a thin flux tube, is a source of discrepancy between the
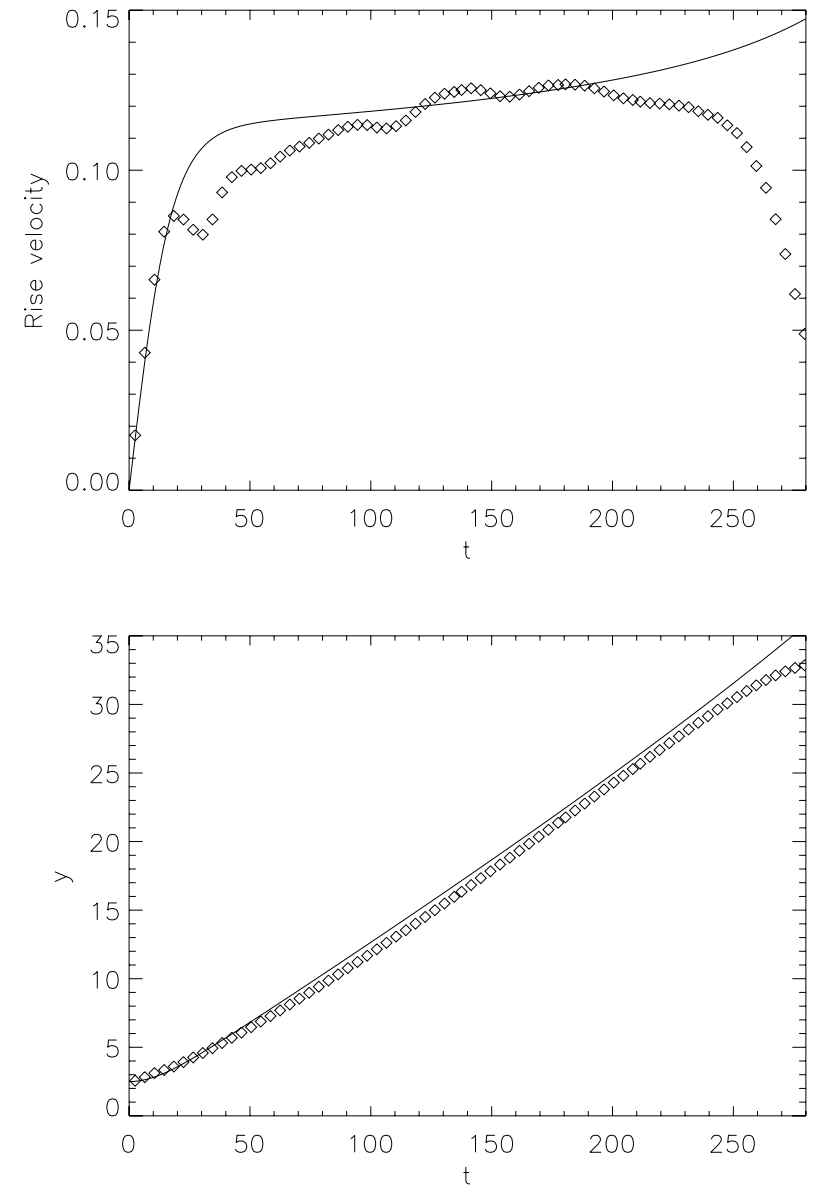

Fig. 8. Rise velocity (upper panel) of the main tube and its height (lower panel), both as functions of time. Diamonds indicate values from the MHD simulation (run A4). The solid line shows the velocity profile calculated with the thin flux tube model, with $C_{D}=2.0$ and $I=2.0$.

simulation results and the thin flux tube calculations. Another discrepancy is the deceleration of the main tube after $t=200$, which is not predicted by the thin flux tube result. This deceleration is due to the closed top boundary condition used. Equation (34) does not take this into account.

Near the top of the simulation domain, the main tube is so large that it can no longer be considered a thin flux tube. At $t=253$, the main tube has an effective radius equal to $H_{p}$. In Sect. 5, we examine in more detail how the limit of the thin flux tube model is reached as the flux tube increases in size.

\subsection{The asymmetric rise of magnetic flux tubes and their trailing vortex streets}

In the simulations discussed thus far, the background atmosphere was initially plane-parallel and the flux tube initially axisymmetric and stationary. This confines the flux tube to a purely vertical trajectory. To study the asymmetric rise of flux tubes, we carried out an additional simulation run. Run B has essentially the same initial condition as Run A4 $\beta_{0}=15$, $\left.\lambda=0.25, R_{0}=0.5\right)$, with the exception that the flux tube 
is initially rotating solidly about its axis with angular velocity $\omega=0.03$. The vorticity integrated over the initial flux tube $\left(r \in\left[0,2 R_{0}\right]\right)$ is $\Omega_{0}=\int_{0}^{2 R_{0}} \nabla \times \boldsymbol{v}_{t} \mathrm{~d} S=\oint v \cdot \mathrm{d} \boldsymbol{l}=0.2$.

As already mentioned in the introduction, a flux tube with a net vorticity $\boldsymbol{\Omega}$ travelling with forward velocity $\boldsymbol{V}$ with respect to the external medium experiences a lift force equal to $-\rho \boldsymbol{\Omega} \times \boldsymbol{V}$. The lift force causes a sideways acceleration of the flux tube so that its motion deviates from the vertical. In simulation runs $\mathrm{A} 1$ to $\mathrm{A} 4$, we have seen that a flux tube rising purely vertically sheds equal but opposite amounts of vorticity to the left and right halves of its wake. When the tube motion is no longer purely vertical, the two sides of the tube shed unequal amounts of vorticity. Each time a vortex roll is emitted from the flux tube, the remaining tube and wake structure is left with a net vorticity.

Emonet et al. (2001) found that the quasi-periodic shedding of vorticity of alternating sign by a rising flux tube leaves this tube and wake structure with a net circulation that reverses its sign periodically in time. Thus the horizontal component of the lift acceleration also alternates periodically. This results in an oscillatory, horizontal motion of the flux tube super-imposed on the general vertical rise of the tube, so that it traces out a zigzag path. By adding the lift acceleration $-\boldsymbol{\Omega} \times \boldsymbol{v}$ to the equation of motion of the thin flux tube and assuming a sinusoidal time-varying vorticity for the tube and wake, they could model the zigzag motion of the tube. We found that $\Omega_{z}(t)=0.04 \sin (2 \pi t / 80+5.2)$ gives a good agreement between the integrated path from the thin flux tube model (plotted as a dashed line in Fig. 9) and the actual path of the main tube. The sequence of circles indicate the positions and effective radii of the main tube as it rises and expands.

Figure 10 shows the vorticity distribution at $t=280$. The three vortex rolls of alternating sign constitute a pattern reminiscent of a von Kármán vortex street. The first vortex roll shed by the flux tube is centered at $(x, y)=(-3,12)$ and has negative sign. This means that, as this roll was being shed, the remaining tube and wake system was gaining a net positive vorticity. The lift force then acts to steer the tube and wake system towards the right. During this time, vortex rolls with positive vorticity are preferentially shed off the tube. This continues until the tube and wake system has a net negative vorticity. At this point, the lift force pushes the tube back towards the left.

\section{Exploring the limits of the thin flux tube approximation}

In this section, we address the question: How relevant are the predictions of the thin flux tube model in describing the quasistatic structure of a rising flux tube as its radius approaches and exceeds the local pressure scale height? To explore the transition from the thin flux tube regime to the "thick" flux tube regime, we performed a simulation (Run C) of a rising tube, starting with a flux tube with an initial flux 100 times larger than in the previous cases. Here, the domain spans $x \in$ $[-40,40]$ and $y \in[0,70]$. The flux tube is initially centered at $\left(x_{0}, y_{0}\right)=(0,20)$ with $B_{0}=12.3, R_{0}=5$ (corresponding to $1 / 4$ of the local pressure scale height) and $\lambda=0.1$.

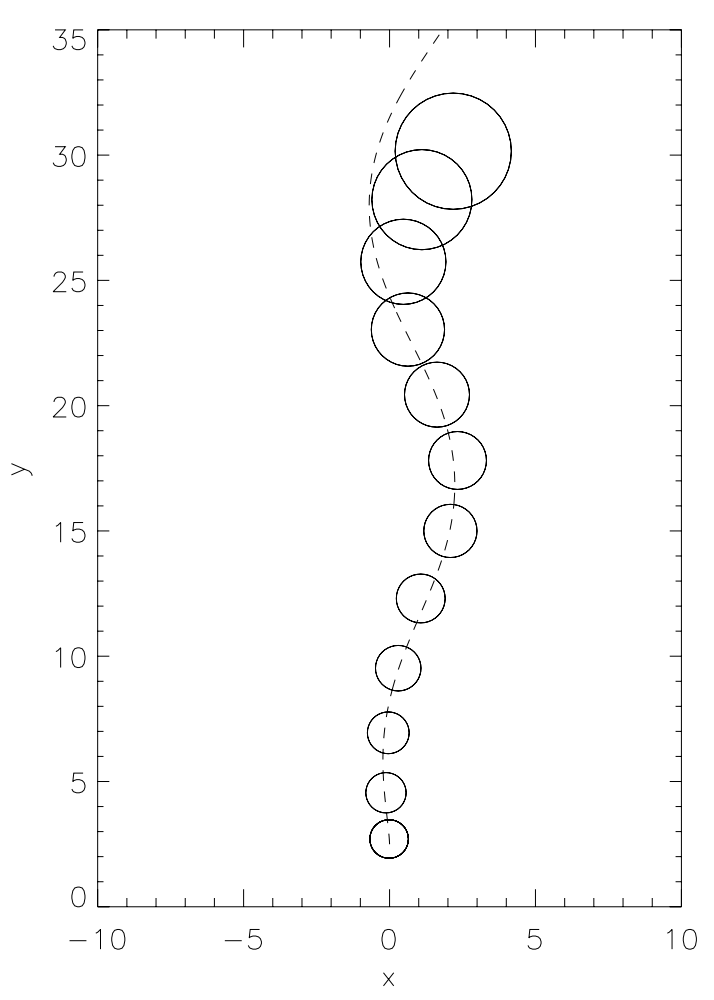

Fig. 9. The trajectory of the main tube in run B. The circles indicate the position of the main tube at different times during the simulation. The effective radius of the main tube at different instances is given by the size of the circles in the plot. The dashed line shows the trajectory from a thin flux tube calculation, taking into account the aerodynamic lift force.

Figure 11 shows the flux tube at $t=0$ and $t=220$. The five concentric green contours in the left panel are the planar projection of different field lines. Each of these contours defines a flux roll. By tracking these contours, we follow the evolution of these flux rolls. The longitudinal flux and initial radius of each of these rolls is given in Table 2 .

In order to evaluate the validity of the thin flux tube approximation, we compare its predictions with the simulation results for all five flux rolls. For the thin flux tube predictions, Eqs. (30)-(33) are used to calculate how the physical quantities evolve. The initial values $\left(B_{0}, \rho_{0}\right.$, etc. $)$ are taken as averages inside the rolls. The values from the thin flux tube calculations are then compared with the average values measured in the flux rolls at later times in the simulation.

Figure 12 shows the relative discrepancies between the model predictions and the average values from the simulation as a function of the effective radius of largest flux roll (flux roll 5). The size of this flux roll is representative of the "true" size of the rising flux tube. As expected, the discrepancy grows as the effective radius of the flux tube becomes comparable to the local pressure scale height. In this regime, the flux tube interior is sufficiently stratified that average values of its physical properties do not match with the corresponding thin flux tube values, so the thin flux tube approximation is no longer appropriate for describing the state of the flux tube. The discrepancies in temperature and density do not grow as much as the discrepancies in field strength and $\beta$ for the following reason: for 


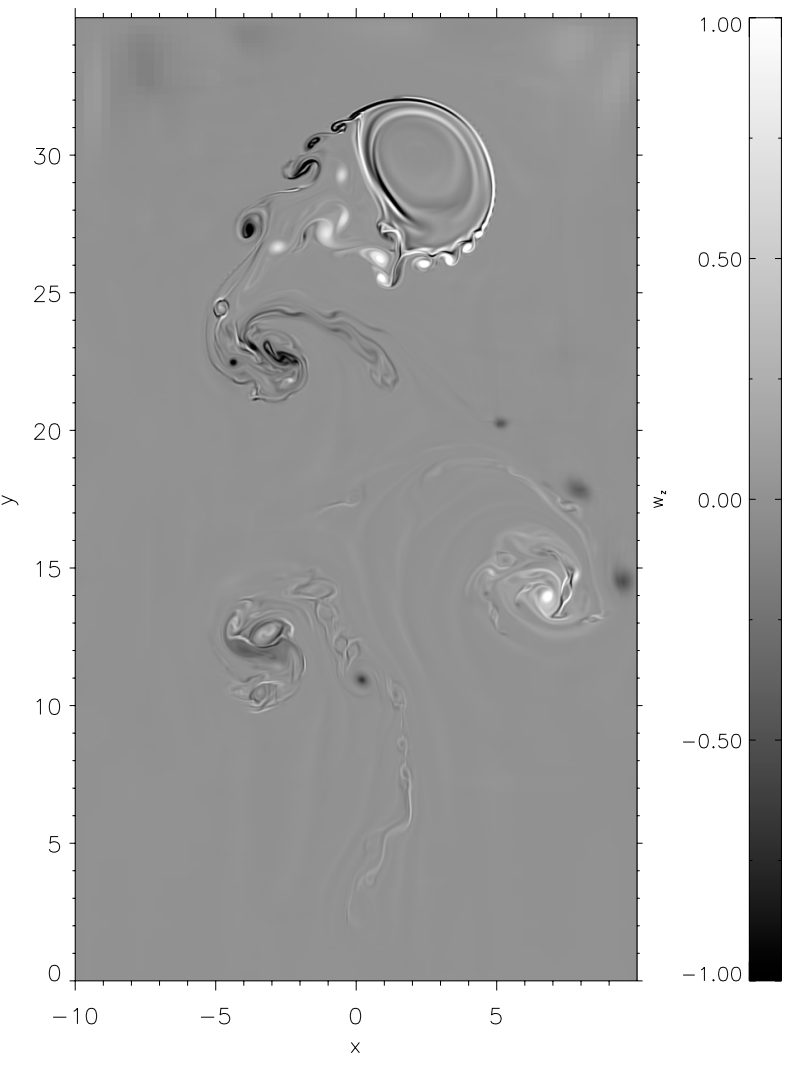

Fig. 10. The distribution of the vorticity at $t=280$ for run B. In this simulation, the flux tube had an initial net vorticity. Aerodynamic lift causes the flux tube to rise in a zigzag fashion, leaving behind a vortex street in its wake.

Table 2. The initial radius and flux of the flux rolls as shown in Fig. 11a.

\begin{tabular}{rccc}
\hline \hline Flux roll & $R\left(H_{p}\right)$ & $\Phi\left(\Phi_{0}\right)$ & Symbol in Fig. 12 \\
\hline 1 & 0.02 & 0.01 & + \\
2 & 0.08 & 0.1 & $\diamond$ \\
3 & 0.1 & 0.2 & $\triangle$ \\
4 & 0.2 & 0.5 & $\square$ \\
5 & 0.3 & 0.8 & $\times$ \\
\hline
\end{tabular}

an ideal thin flux tube in pressure balance with its surroundings, the relative difference of $T$ and $\rho$ between the tube and its surroundings is always of order $O\left(\beta^{-1}\right)$. Thus relative differences in $T$ and $\rho$ decrease with increasing $\beta$ (the case of an expanding tube). Consequently, discrepancies in $T$ and $\rho$ between the thin tube predictions and the simulation results do not tell whether the thin flux tube approximation is good at describing the average properties of the flux tube. In contrast, discrepancies in $\beta$ and $|B|$ show clearly the transition between the thin flux tube regime to the "fat" flux tube regime, when the radius of the tube is comparable to the pressure scale height.

We have also examined how the average twist, $\langle\lambda\rangle$, of each flux roll evolves as they expand. Figure 13 shows $\langle\lambda\rangle$ as a function of the effective radii of the flux rolls. Again, there is good
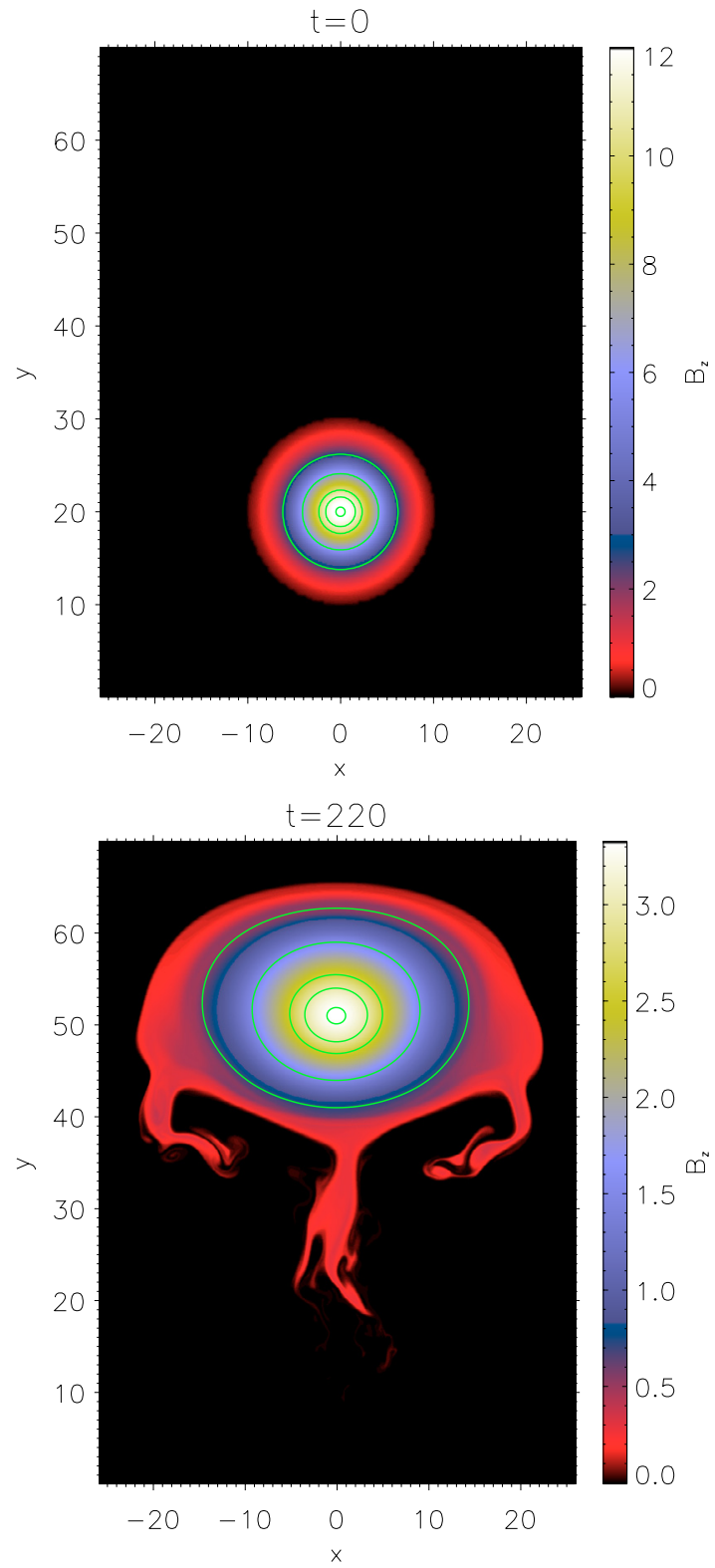

Fig. 11. Evolution of a large flux tube (Run C). Shown in color is the longitudinal field. Each of the five circular green contours represent a magnetic field line projected onto the plane. Every contour encloses a certain amount of flux, which defines a flux roll. Table 2 gives the flux and initial radius of each flux roll.

agreement between the data points and the solid curve, which plots the relation given by Eq. (21).

\section{Conclusions}

We have carried out idealized 2.5D MHD simulations of buoyant magnetic flux tubes rising in a stratified layer over a range of Reynolds numbers $(25 \gtrsim \operatorname{Re} \gtrsim 2600$ ). Our simulations confirm previous results in the literature. Additionally, we have analyzed the dependence of the results on the Reynolds number. We found that the detailed structure of the wake, as well as the amount of flux retained in the main tube, varies with the Reynolds number. At sufficiently high Reynolds 

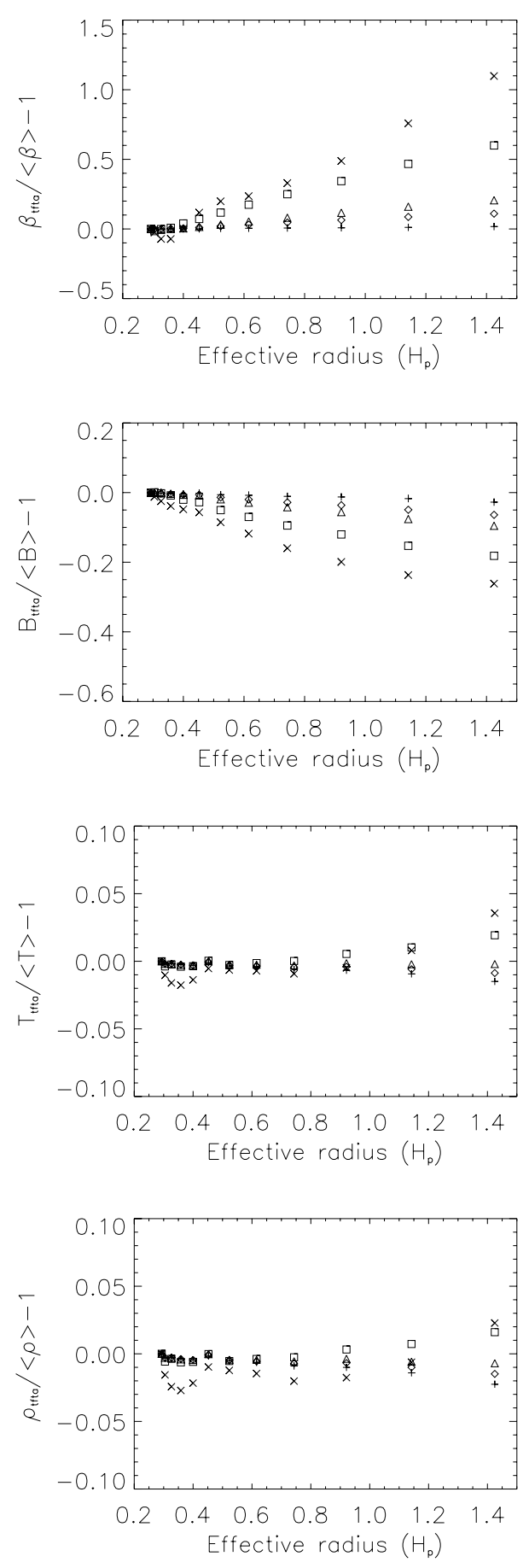

Fig. 12. Comparison between thin flux tube calculations with average quantities inside the flux rolls (Run C). The different symbols correspond to quantities in the different flux rolls (see Table 2). As the effective radius of the flux tube approaches the local pressure scale height $H_{p}$, the discrepancy between the simulation results and the thin flux tube predictions grows.

number ( $\operatorname{Re} \gtrsim 600$ ), the vortex pair in the wake breaks into secondary rolls. The amount of flux retained in the tube also increases with Re.

We have studied how the twist in a flux tube varies as it expands. In particular, the dimensionless twist $\langle\lambda\rangle$, averaged over the main tube, scales approximately linearly with the tube

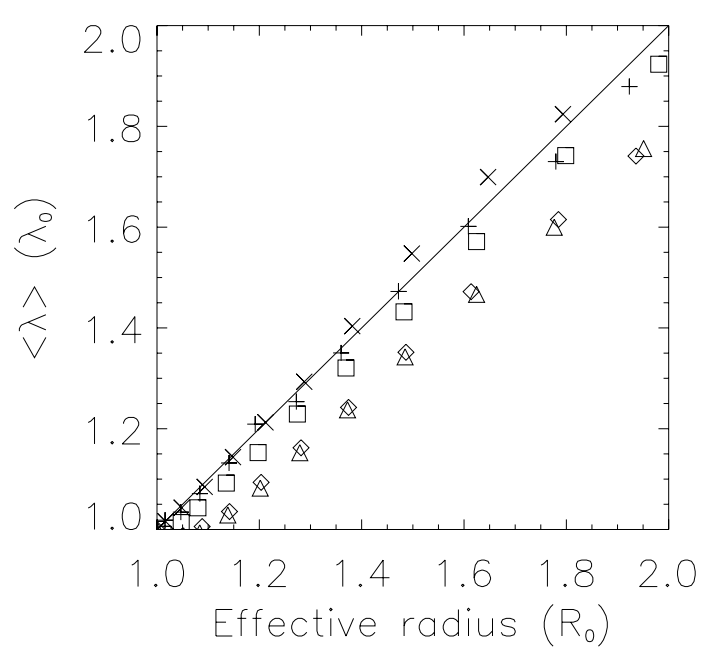

Fig. 13. Variation of the average twist of the flux rolls as a function of their effective radii. The different symbols show the average twist of the five flux rolls (Run C). The solid line shows the relation given by Eq. (21). The good match between the simulation results and relation (21) indicates that the flux rolls expand homologously.

radius. If flux tubes originating from the bottom of the solar convection zone have any amount of initial twist, this twist will be amplified upon the rise of the tube. The twist will be maximum at the apex of the rising loop, where the cross-sectional radius is largest.

We derived thin flux tube equations (Eqs. (30)-(33)) to model the evolution of the properties of a horizontal flux tube rising adiabatically through the atmosphere. Using these equations to model the expansion of the tube, and using Eq. (34), the motion of the main tube can be reproduced. For a tube that undergoes asymmetric vortex shedding, the lift force can be included into the equation of motion to explain the zigzag motion of the tube. The vortex shedding associated with this zigzag motion leaves behind a vorticity distribution resembling a von Kármán vortex street.

Furthermore, we studied the transition from the regime of thin flux tubes to the regime of fat flux tubes (Run C). We found that the discrepancy between the thin tube calculations and the average quantities of flux rolls increases as the flux tube expands. When its radius is comparable to one pressure scale height, the discrepancy between the thin tube calculations and the simulation results can be at least of order $O(1)$, meaning the thin flux tube approximation is no longer valid. This result is in accordance with previous expectations. In the solar convection zone, rising toroidal flux tubes approach this limit at a depth of about $10 \mathrm{Mm}$, so it is no longer appropriate to continue thin flux tube simulations above those depths. On the other hand, our results point in the direction that below such depths, the thin flux tube approximation is useful for studying the evolution of flux tubes.

Acknowledgements. We would like to thank the anonymous referee for helpful comments which improved the presentation of the results. MCMC acknowledges financial support from the International Max Planck Research School at the Max Planck Institute for Solar System Research. 


\section{References}

ASCI/Alliance Center for Thermonuclear Flashes 2003, FLASH 2.3 User Manual, University of Chicago http://flash.uchicago.edu

Batchelor, G. 1967, An Introduction to Fluid Dynamics (Cambridge: Cambridge University Press)

Caligari, P., Moreno-Insertis, F., \& Schüssler, M. 1995, ApJ, 441, 886

Chandrasekhar, S. 1957, An introduction to the study of stellar structure (New York: Dover Publications)

D'Silva, S. Z., \& Choudhuri, A. R. 1991, Sol. Phys., 136, 201

Emonet, T., \& Moreno-Insertis, F. 1998, ApJ, 492, 804

Emonet, T., Moreno-Insertis, F., \& Rast, M. P. 2001, ApJ, 549, 1212

Fan, Y. 2004, Living Rev. Sol. Phys., 1, 1

http: //www. livingreviews.org (cited on 8/11/2005)

Fan, Y., Fisher, G. H., \& Deluca, E. E. 1993, ApJ, 405, 390

Fan, Y., Fisher, G. H., \& McClymont, A. N. 1994, ApJ, 436, 907

Fan, Y., Zweibel, E. G., \& Lantz, S. R. 1998a, ApJ, 493, 480

Fan, Y., Zweibel, E. G., Linton, M. G., \& Fisher, G. H. 1998b, ApJ, 505, L59

Ferriz-Mas, A., Schüssler, M., \& Anton, V. 1989, A\&A, 210, 425
Hughes, D. W., \& Falle, S. A. E. G. 1998, ApJ, 509, L57

Hughes, D. W., Falle, S. A. E. G., \& Joarder, P. 1998, MNRAS, 298, 433

Linton, M. G., Longcope, D. W., \& Fisher, G. H. 1996, ApJ, 469, 954

Longcope, D., Fisher, G., \& Arendt, S. 1996, ApJ, 464, 999

Magara, T. 2001, ApJ, 549, 608

Moreno-Insertis, F. 1997, Mem. Soc. Astron. It., 68, 429

Moreno-Insertis, F., \& Emonet, T. 1996, ApJ, 472, L53

Moreno-Insertis, F., Caligari, P., \& Schüssler, M. 1994, Sol. Phys., 153, 449

Parker, E. N. 1974, ApJ, 191, 245

Parker, E. N. 1975, ApJ, 198, 205

Parker, E. N. 1979, Cosmical magnetic fields: Their origin and their activity (Oxford: Clarendon Press; New York, Oxford University Press), 858

Roberts, B., \& Webb, A. R. 1978, Sol. Phys., 56, 5

Schüssler, M. 1979, A\&A, 71, 79

Spruit, H. C. 1981, A\&A, 98, 155

Toro, E. L. 1997, Riemann Solvers and Numerical Methods for Fluid Dynamics (Berlin Heidelberg: Springer-Verlag) 